\title{
Presenting With Status Epilepticus That Neurosyphilis: Case Report
}

\author{
Aylin Bican Demir ${ }^{\mathrm{a}, \mathrm{c}}$, Ibrahim Bora ${ }^{\mathrm{a}}$, Ozlem Taskapilioglu ${ }^{\mathrm{a}}$, Necdet Karli ${ }^{\mathrm{a}}$, Bahattin Hakyemez
}

\begin{abstract}
Syphilis; which is a sexually transmitted disease that is caused by the agent Treponema Pallidum has been seen less frequently with penicillin therapy. In recent years, with the emergence of HIV infection and the increasing number of patients with immune deficiency, syphilis has started being seen frequently and gained importance. If the patients with primary and secondary syphilis are not treated, approximately $7-9 \%$ of them may develop neurosyphilis. Meningovasculary syphilis is characterized with conditions such as hemiparesis, aphasia, visual loss, confusion that are caused by cortical and subcortical infarcts. Thirty-six year old male and 35-year-old male patients presented to our hospital with status epilepticus. We decided to discuss neurosyphilis cases with literature that presented with generalized seizures, mental confusion, hemiparesis and had MRI findings compatible with herpes simplex encephalitis and lesions that disappeared with antibiotic therapy.
\end{abstract}

Keywords: Neurosyphilis; Status Epilepticus; MRI

\section{Introduction}

Syphilis; which is a sexually transmitted disease that is caused by the agent Treponema Pallidum has been seen less frequently with penicillin therapy. In recent years, with the emergence of HIV infection and the increasing number of patients with immune deficiency, syphilis has started being seen frequently and gained importance. Nowadays, the ex-

Manuscript accepted for publication April 18, 2012

${ }^{a}$ Department of Neurology, Uludag University Medical Faculty, Turkey ${ }^{\mathrm{b}}$ Department of Radiology, Uludag University Medical Faculty, Turkey ${ }^{c}$ Corresponding author: Aylin Bican Demir, Uludag University Medical School Neurology, Uludag Universitesi Tip Fakultesi, Noroloji ABD, 16059/Gorukle, Bursa, Turkey. Email: aylinbd@uludag.edu.tr

doi:10.4021/jnr102w act incidence of syphilis patients is unknown. If the patients with primary and secondary syphilis are not treated, approximately $7-9 \%$ of them may develop neurosyphilis [1].

The first clinical manifestation of neurosyphilis that is seen in approximately $25 \%$ of all syphilis cases is usually asymptomatic meningitis [2]. Meningovasculary involvement is also seen in early stage (first $1-12$ years) neurosyphilis. Meningovasculary syphilis is characterized with conditions such as hemiparesis, aphasia, visual loss, confusion that are caused by cortical and subcortical infarcts. Tabes dorsalis, optic atrophy and meningomyelitis develop in the late stage. Meningoencephalitis develops approximately 15 to 20 years later. At this stage, behavioral changes, memory and mood disorder, partial and generalized seizures, dementia occurs and if not treated death occurs within 3 - 5 years [2]. In one study, the frequency of seizures in patients with neurosyphilis varies between 14 to $60 \%$ [3].

Syphilis is a disease that can involve all the organs and tissues in the body and present with many different clinical manifestations; we decided to discuss neurosyphilis cases with literature that presented with generalized seizures, mental confusion, hemiparesis and had Magnetic Resonans Imaging (MRI) findings compatible with herpes simplex encephalitis and lesions that disappeared with antibiotic therapy.

\section{Case Report}

\section{Case 1}

Thirty-six years old male patient presented to our hospital with status epilepticus. Without any history of systemic diseases, the patient described numbness in the right half of the body for 4 - 5 times in the preceding year and he had a generalized seizure 2 months ago. He was hospitalized and examined by the doctor he resorted to and Carbamazepin 800 $\mathrm{mg} /$ day po. was started and the cranial tomography taken in that period was detected as normal. The patient had generalized tonic-clonic seizure and weakness in the right arm a day before and with developing of mental confusion he was brought to the emergency room. The patient was admitted to 


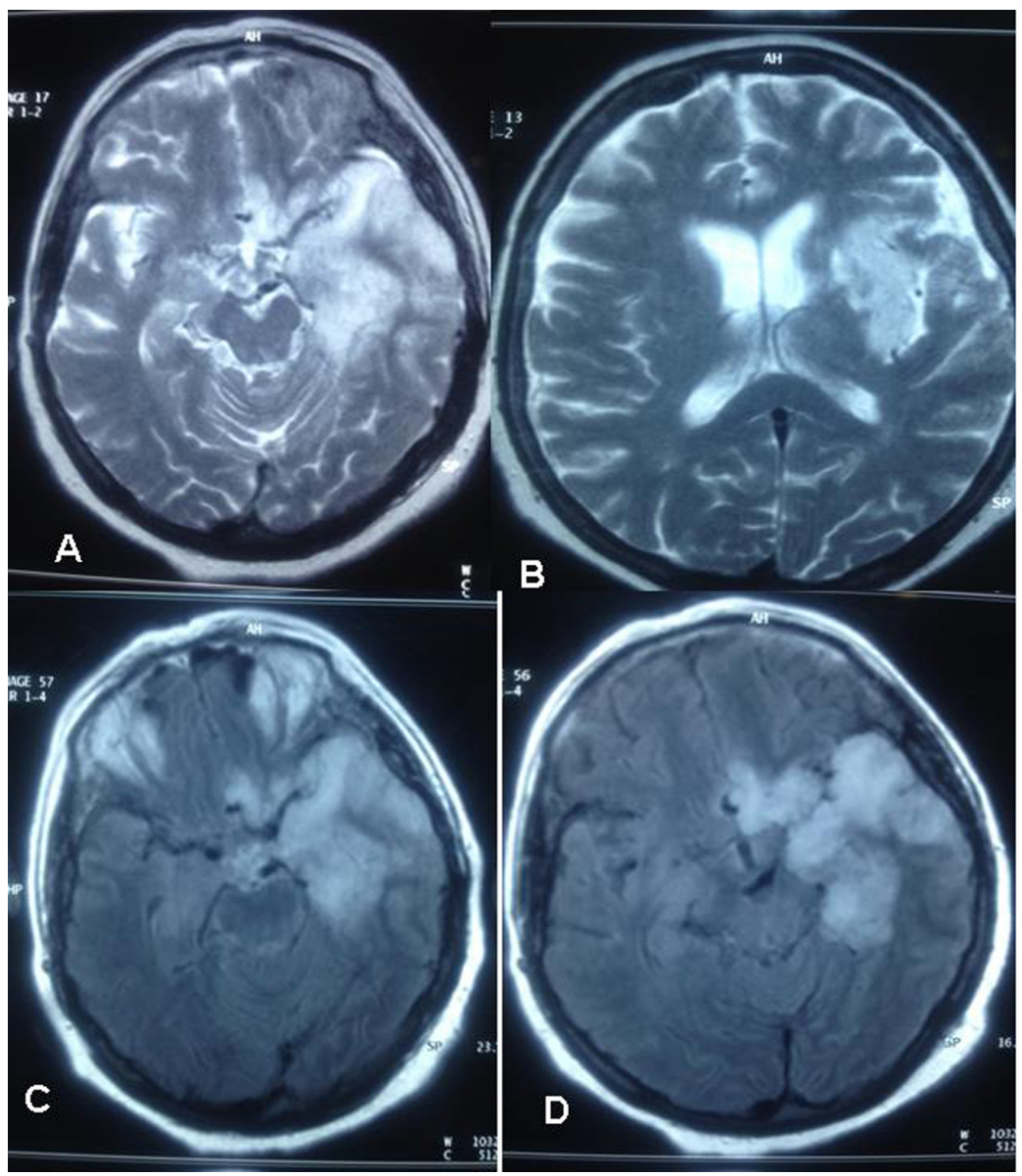

Figure 1. Cranial MRI, lesions were seen more clearly in the left temporal region and as increased intensity in hypothalamus and insula region.

our clinic with a provisional diagnosis of Cerebral Vasculary Disease? Encephalitis? In the performed difusion and cranial MRI, lesions were seen more clearly in the left temporal region and as increased intensity in hypothalamus and insula region (Fig. 1). These lesions that were not compatible with arterial vessel irrigation area were interpreted as encephalitis by the radiology department. The patient was consulted to infectious diseases department with a provisional diagnosis of Encephalitis and leukocytosis, fever, neck stiffness. Herpes encephalitis was thought and acyclovir was started. With the possibility of limnic encephalitis due to paraneoplastic syndrome paraneoplastic panel was sent and came back negative. In the performed Lumbar Puncture 30 luekocytes, 360 erythrocytes were found and in the Cerebro spinal fluid (CSF) biochemical examination protein was $134 \mathrm{mg}$. ANA, anti-nDNA, ANA profile, serum protein electrophoresis and tumor markers were normal. In the performed electroencephalography (EEG)'s of the patient in regular intervals; localized to left periodic lateralized epileptiform discharges (PLED) activity was detected and left hemisphere was interpreted as assisting a destructive event (Fig. 2). RPR-VDRL was positive at $1 / 2$, Syphilis IHA at $1 / 40$, CSF Syphilis IHA at $1 / 28$ and Blood Syphilis at 1/640. The department of Infectious Diseases evaluated the patient's condition and recommended crystallized penicilin for at least 3 weeks. Acyclovir was stopped at the day 14. Clonic contractions were observed in the patient's right hand. Lamictal was added to the antiepileptic therapy and Trileptalin was planned to be stopped slowly. Patient's general condition has improved gradually. Patient's oral intake has improved on the 6th day of crystallized penicillin therapy and the patient start to be mobilized. The patient's consciousness was clear but he was unable to cooperate and talking nonsense. He was assessed by Cardiology in terms of aortitis and his electrocardiography was normal, follow-up was recommended. In terms of neurosyphilis eye involvement, an Optic Coherens Tomography (OCT) was performed on the patient with an Ophthalmology consultation for an optic nevre pathology and nothing was detected. The last neurological examination following findings was existing: predominantly sensorial mixed aphasia, frustrated monoparesis of right lower extremity and indifferent babinski on the right. Last treatment: Topamax 


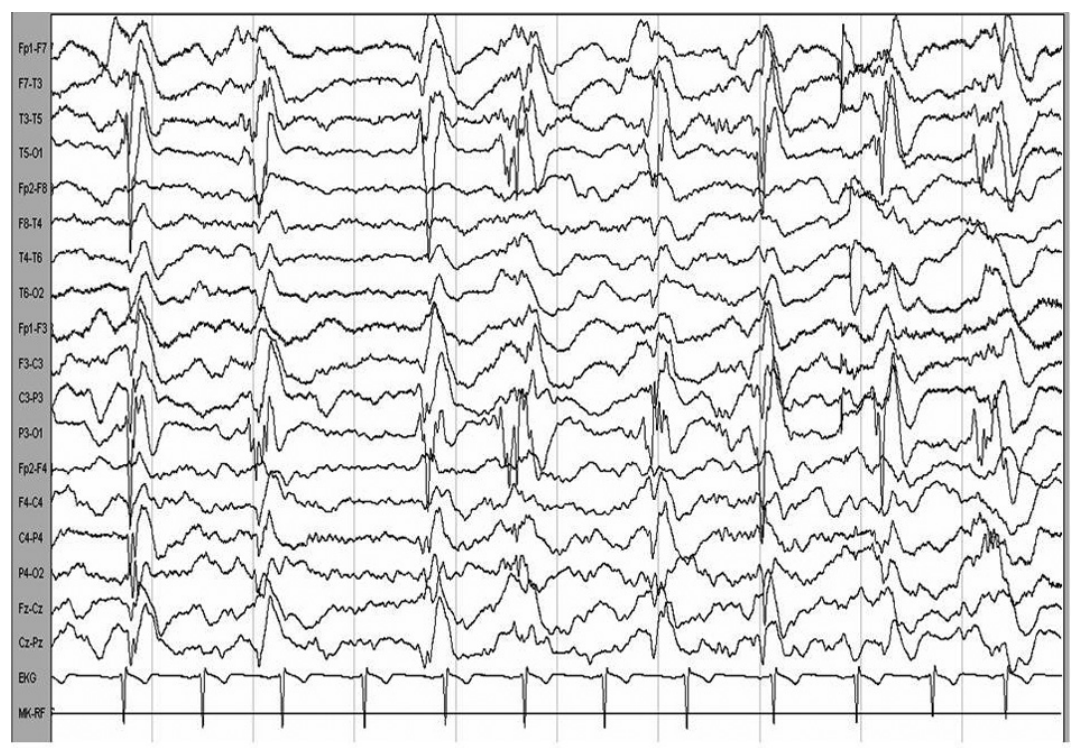

Figure 2. Localized to left periodic lateralized epileptiform discharges (PLED) activity.

$50 \mathrm{mg}, 2 \times 1$.

\section{Case 2}

A 35-year-old male patient who first had a secondary generalized seizure in November, 2000; was diagnosed as epilepsy and started on Tegretol $400 \mathrm{mg} 2 \times 1$. The patient who had seizures and agitated behavior was admitted to our clinic in January, 2001 with a provisional diagnosis of encephalitis. The patient had right hemiparesis and a seizure that lasted about 2 hours on the follow-up, the seizures were controlled with the status protocol. In the performed cranial MRI, increased intensity clear on right temporal and limbic structures and on both hemispheres and on the Single-photon emission computed tomography (SPECT) decreased perfusion was detected on both frontotemporal regions and bilateral medial temporal cortex. In the performed lumbar puncture CSF protein was $50 \mathrm{mg} / \mathrm{dL}$, Syphilis IHA CSF was $1 / 40$ positive and blood IHA was 1/1520 positive. The patient received 24 million units/day Penicillin therapy for about 10 days with a diagnosis of neurosyphilis, syphilis markers remained positive on the follow-up and the patient received tetradox $2 \times 100$ $\mathrm{mg}$ for 14 days and Amoksina $3 \mathrm{gr}$ for 14 days. The patient was followed up with Tegretol $400 \mathrm{mg} 2 \times 1$ for seizures and no significant improevement was seen on the EEG controls (findings compatible with encephalopathy clear on the right hemisphere and mild on the left). The patient had significant amnesia on the follow-ups and depending on the neuropsychologic evaluation his memory tests were impaired. The patient was not followed up regularly and with having frequent complex partial seizures, he was admitted to the clinic in March, 2009. He was receiving Trileptal 600 mg 2 x 1

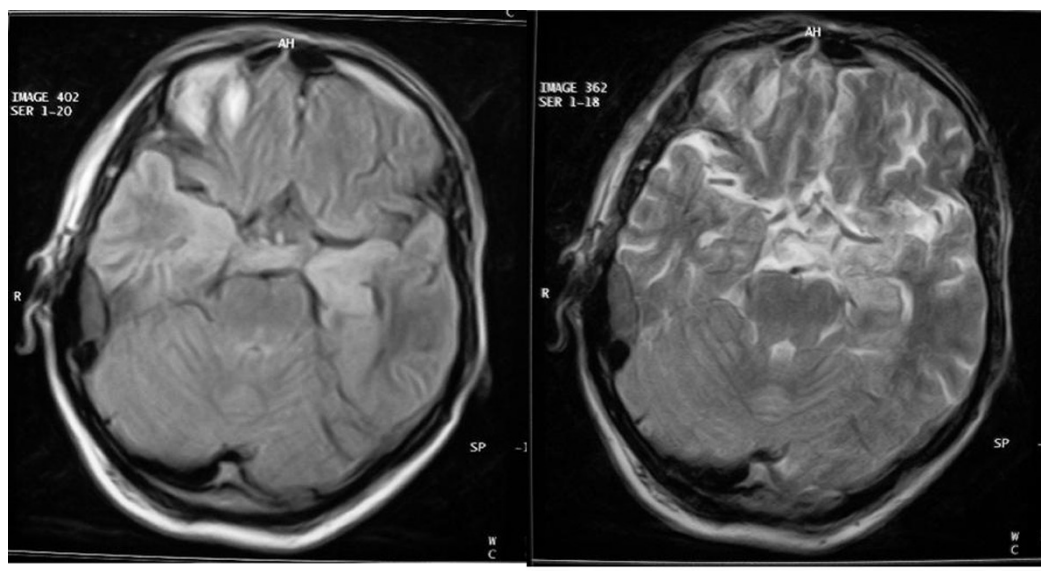

Figure 3. Cranial MRI, increased symmetric intensity was seen on both hippocampal regions. 


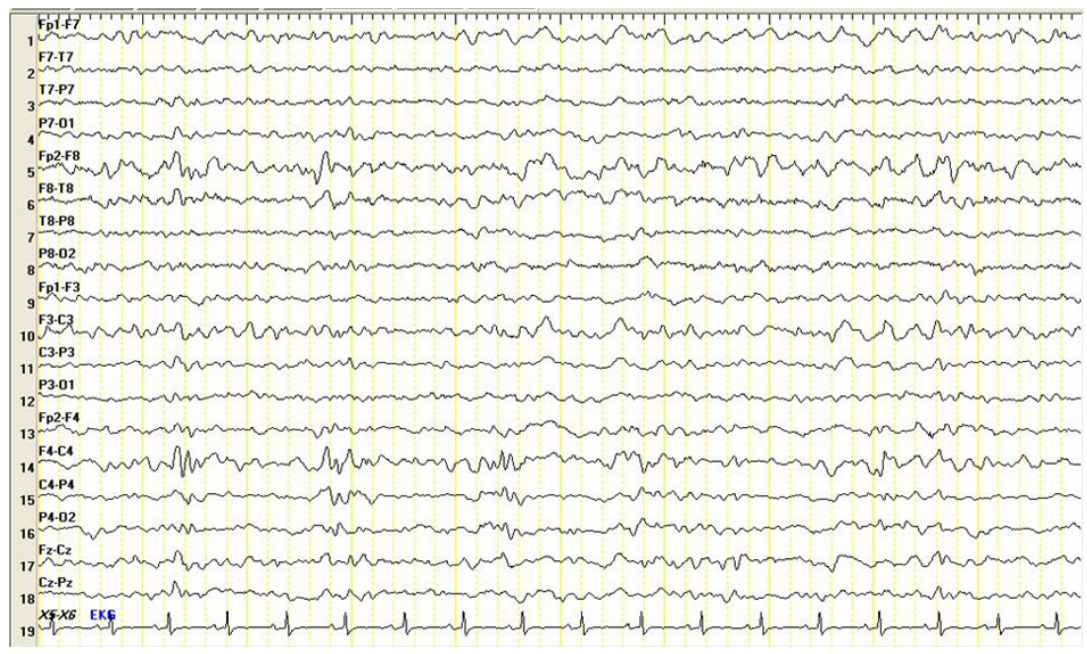

Figure 4. Temporary delta activity on EEG.

and after increasing the doses the seizures were seen to decrease significantly. VDRL-RPR was $(+)$ and Syphilis IHA was $1 / 40(+)$. On the performed NPT, the patient had lack of attention and diffuse and mild difficulty was detected on the memory tests. In the cranial MRI, increased symmetric intensity was seen on both hippocampal regions (Fig. 3). CSF biochemistry and cell count was normal, CSF syphilis IHA at $1 / 28$, Blood syphilis IHA at $1 / 1520$ and RPR was positive at $1 / 4$. The patient was thought to have tertiary syphilis at active stage and started with penicilin $4 \times 2$ million units fort he first day and $4 \times 5$ million units fort he next 3 weeks. He had temporary delta activity on EEG (Fig. 4). He was assessed in terms of cardiac involvement and on the performed Electrocardiography (ECO), no syphilic involvement was found. He was also assessed in terms of eye involvement and no retinitis, choroiditis and optic atrophy was detected.

\section{Discussion}

In the young patients who present with seizures, stroke, mental confusion and dementia; encephalitis, vasculitis, venous thrombosis and paraneoplastic limbic encephalitis are the diseases that are needed to be thought in the first plan [2]. A patient of ours had seizures, right hemiparesis and mental confusion; and the brain MRI showed signal changes on both hypothalamus and insula regions, being more clearly on left temporal region and herpes simplex encephalitis was thought in the first place. CA-125, CA 19-9, Ca 15-3, AFP, CEA markers and anti-Hu, anti-yo, anti-Yi antibodies were assessed in terms of paraneoplastic limbic encephalitis and the results were negative. We excluded collagen tissue diseases because ANA profile was negative and there were no systemic findings or kidney involvement. VDRL and FTAabs values were positive in the serum and CSF. Our second patient presented with status epilepticus. There is not a golden standard method for definitive diagnosis of neurosyphilis. The diagnosis is made based on the clinical presentation and supportive laboratory findings [2]. The therapy protocol on neurosyphilis cases depending on the guideline that was published in 2002 is $12-24$ million units/day of i.v. crystallized penicillin for $10-14$ days. The alternative therapy is 2.4 million units of intramusculary procain penicillin and Probenesid 4 x125 mg. po. Tetracycline, chloramphenicol and ceftriaxone is used in patients with penicillin allergy [4], $4 \times 6$ million units of crystallized penicillin was used for 14 days in our cases. Our patient showed indolent improvement after the treatment.

Neurosyphilis is recently seen with HIV infection with an increased frequency. In a case with HIV positive neurosyphilis of Kyebamba the patient presented with left hemiparesis and Jacksonian seizure and it was suggested that in patients with neurosyphilis personality changes in $33 \%$, ataxia in $28 \%$, stroke in $23 \%$, eye findings in $17 \%$, urinary system findings in $17 \%$, headache in $10 \%$ and seizures in $7 \%$ may be seen. In cranial MRI's of the patients of Gurses et al followed with a diagnosis of status epilepticus in 2006, significant temporal lobe involvement on one side with cerebral atrophy was found and the etiology was neurosyphilis in 3 HIV negative patients. In recent years, neurosyphilis cases that make signal changes on temporal lobes and have lesions that disappear with antibiotic treatment are being seen frequently. There are studies that suggest subcortical ischemic foci, atrophy on the cerebellum and the brain stem and diffuse infarcts in cranial MRI in neurosyphilis [5]. In studies that are made with neurosyphilis patients involvement of one side temporal lobe in radiologic evaluation and in a case in Taiwan, involvement of both sides of temporal lobes was reported to literature $[6,7]$.

We decided to discuss neurosyphilis cases with literature 
that presented with generalized seizures, mental confusion, hemiparesis and had MRI findings compatible with herpes simplex encephalitis and lesions that disappeared with antibiotic theropy.

\section{References}

1. Bharucha NE, Ramamoorthy K, Sorabjee J, Kuruvilla T. All that caseates is not tuberculosis. Lancet. 1996;348(9037):1313.

2. Rowland LP, Stefanis L: Merritt's neurology, Spirochete infections 11. Edition. Lippincott Williams and Wilkins, New York, p. 235-242, 2005.

3. Hooshmand H. Seizure disorders associated with neuro- syphilis. Dis Nerv Syst. 1976;37(3):133-136.

4. Gilbert DN, Treatment of neurosyphilisis in the Sanford Guide to antimicrobial teraphy 2002.

5. Hussein R, Fisher M, Rai AT. A case of neurosyphilis involving the cerebellum on magnetic resonance imaging (MRI) with resolution of the abnormality after treatment. Int J Infect Dis. 2008;12(1):103-105.

6. Vieira Santos A, Matias S, Saraiva P, Goulao A. Differential diagnosis of mesiotemporal lesions: case report of neurosyphilis. Neuroradiology. 2005;47(9):664-667.

7. Chung-Wen-Chen . General paresis with reversible mesial temporal T2-weighted hyperintensity on mahnetic resonans image: A case report. Acta Neurology Taiwan 2005: $14 ; 208-212$ 